\title{
Sechs Achsen der Problemoptik
}

In meiner Literaturrecherche fiel mir auf, dass sich in der Diskussion über das hard problem verschiedene Optiken zeigen, die sich als bipolare Achsen des Problemzuganges kategorisieren lassen. Die jeweilige Problemoptik bestimmt zu einem hohen Grade die Art des Lösungsversuches. Bevor ich auf den für diese Arbeit zentralen Problemzugang der phänomenalen Achse eingehe, werde ich in den folgenden Abschnitten kurz auf alle sechs, von mir festgestellten Optiken eingehen. Selbstverständlich erhebe ich nicht den Anspruch auf eine Vollständigkeit dieser Kategorisierung. Es ist zudem wichtig zu bedenken, dass die Fronten dieser bipolaren Achsen zwischen einzelnen Vertretern bzw. Ansätzen nicht gleichmässig verlaufen. Das heisst, dass phänomenale Realisten, die bspw. bezweifeln, dass Erleben mit einem reduktiven Funktionalismus zufriedenstellend erklärt werden kann (explanatorischer Kritizismus), zwar epistemisch pessimistisch $^{1}$ (z.B. McGinn) ${ }^{2}$ sein können, aber dennoch nicht santimaterialistisch oder substanzdualistisch argumentieren müssen. ${ }^{3}$

Ein anderes Beispiel für die Unregelmässigkeit in der Verteilung der Antipoden ist, dass Panpsychismus oder neutraler Monismus Phänomenales zwar ernst nehmen (phänomenaler Realismus) und Erleben als eigenständiges Explanandum anerkennen. Aus diesem Grund behaupten sie nicht, wie der materialistische Monismus, dass Phänomenales `letztlich $<$ nur etwas Materielles sei. Dennoch müssen panpsychistische Vertreter eines phänomenalen Realismus nicht zwingend und im engen Sinn >immaterialistisch< bzw. >antiphysikalistisch oder >antinaturalistisch $<$ argumentieren, falls sie sich auf einen neutralen Monismus berufen. Im Rahmen dieser Dissertation kann ich jedoch nur skizzenhaft auf jene Achsen von Problemzugängen eingehen. Weil der Fokus meiner Arbeit auf dem Desiderat phänomenaler Adäquatheit liegt, werde ich nur auf die letzte der sechs folgenden Achsen näher eingehen.

1 Unter epistemischem Pessimismus verstehe ich die Ansicht, dass es weder mit reduktiven noch mit nicht-reduktiven Ansätzen möglich ist, Bewusstsein zu erklären.

2 Vgl. McGinn, Colin: »Can We Solve the Mind-Body Problem?«, Mind (New Series), [1989] Vol. 98 , No. 391, S. 36o.

3 So vermutet bspw. McGinn trotz seiner mysterianistischen Position, dass Bewusstsein »[...] in irgendeiner Weise an die Natur von Materie gebunden ist «. McGinn, Colin: Wie kommt der Geist in die Materie? Das Rätsel des Bewusstseins, München: Beck 2001, S. 233. Die Weise, wie Bewusstsein an Materie gebunden, ja sogar mit ihr eine »unlösbare Einheit« bilde, sei jedoch eine $»[\ldots]$ Weise, die wir nicht verstehen $[\ldots]$... A.a.O., S. $25^{2}$. 
Diese Achse der Problemoptik besteht in Kontroversen zwischen diversen Formen von Dualismen resp. Monismen. Die Varianten von Dualismen (bzw. Monismen) lassen sich durch die Frage bestimmen, im Hinblick auf welche Grösse man (nicht) dualistisch denkt. ${ }^{4}$ Im Hinblick auf das hard problem besteht die Problemoptik dieser Achse aus ontologischen Fragen darüber, was mentale und physikalische Zustände >letztlich sind resp. ob eine Zustandsklasse eine Unterform der anderen ist oder nicht. ${ }^{5}$

Dualistische Konzeptionen beschränken sich jedoch nicht auf einen klassischen, substanzdualistischen Cartesianismus ${ }^{6}$ bzw. zeitgenössischen Substanzdualismus, wie z.B. denjenigen von Swinburne ${ }^{7}$ oder Foster. ${ }^{8}$ Konzeptionen auf der dualistischen Seite der Ontologischen Problemoptik können auch in Eigenschaftsdualismen oder - als schwächste Form des Dualismus - in einem Prädikatendualismus bestehen. ${ }^{9}$ Psychologische oder mentale Prädikate sind nach dem Prädikatendualismus für eine vollständige Beschreibung unserer Welt unabdingbar. ${ }^{10}$ Anders gesagt: mentale Prädikate sind nach ihm nicht auf physikalische Prädikate reduzierbar. ${ }^{11}$ So basiert die Konzeption der

4 Vgl. Robinson, Howard: »Dualism«, in: The Stanford Encyclopedia of Philosophy, 2013, Online: http://plato.stanford.edu/archives/fall2o13/entries/dualism/ [zuletzt geprüft am 25.09.2018], Abschnitt 2.0.

$5 \quad$ Vgl. a.a.O., Abschnitt 1.1.

6 Der cartesianische Dualismus besteht nach Seager in der Weigerung, mentale Phänomene in ein naturwissenschaftliches Weltbild einer physikalischen Welt zu integrieren. Stattdessen werden Materie und Geist als mehr oder weniger unabhängige Domänen der Interaktion betrachtet. Seager, William / Allen-Hermanson, Sean: »Panpsychism«, in: The Stanford Encyclopedia of Philosophy, 2013, Online: http://plato.stanford.edu/archives/ fall2013/entries/panpsychism/ [zuletzt geprüft am 25.09.2018], Abschnitt 1.o.

Vgl. Swinburne, Richard: »Body and Soul«, in: The mind-body problem. A guide to the current debate, Warner, Richard / Szubka, Tadeusz [Hrsg.], Cambridge, Massachusetts: Blackwell 1994, S. 311-316.

8 Vgl. Foster, John: The immaterial self. A defence of the Cartesian dualist conception of the mind, London: Routledge \& Kegan Paul 2005. Vgl. dazu auch Van Gulick, Robert: »Consciousness «, in: The Stanford Encyclopedia of Philosophy, 2014, Online: http://plato. stanford.edu/archives/spr2014/entries/consciousness/ [zuletzt geprüft am 17.09.2018], Abschnitt 8.1.

9 Vgl Robinson, Howard: »Dualism«, in: The Stanford Encyclopedia of Philosophy, 2013, Online: http://plato.stanford.edu/archives/fall2013/entries/dualism/ [zuletzt geprüft am 25.09.2018], Abschnitt 2.1.

10 Vgl. ebd.

11 Konkret und im Hinblick auf die Problemoptik zum hard problem heisst das, dass bei einem Prädikatendualismus mindestens darauf bestanden wird, dass Mentales mit "geist-unabhängigen Begriffen« (»mind-independent terms«) aus der Physik weder 
Irreduzibilität von Spezialwissenschaften, wie z.B. die Nicht-Reduzierbarkeit der Psychologie auf Physik, (mindestens) auf einem Prädikatendualismus. ${ }^{12}$

Nach Robinson ist der Eigenschaftsdualismus eine leicht stärkere Form des Dualismus als der Prädikatendualismus. Denn letzterer geht primär von einer sprachlichen Nicht-Reduzierbarkeit aus, die nicht unbedingt ontologische Konsequenzen haben muss. Der Eigenschaftsdualismus geht jedoch von einem essenziellen bzw. ontologischen Unterschied zwischen mentalen und physikalischen Eigenschaften aus. ${ }^{13}$

Schon fast ${ }^{14}$ in der Mitte dieser Achse liegen Positionen des dualen AspektMonismus (dual-aspect monism), für die Geist und Materie zwar irreduzibel sind, sich aber beide Aspekte auf eine, ihnen zugrunde liegende »neutrale Domäne ${ }^{15}$ zurückführen lassen. ${ }^{16}$ In der Mitte zwischen Dualismen und Monismen liegen Positionen des neutralen Monismus ${ }^{17}$ oder des reflexiven Monismus, ${ }^{18}$ für die, vereinfacht gesagt, sowohl physikalische als auch

vollständig beschrieben noch erklärt werden kann. Warner, Richard: »Introduction: The Mind-Body Debate«, in: The mind-body problem. A guide to the current debate, Warner, Richard / Szubka, Tadeusz [Hrsg.], Cambridge, Massachusetts: Blackwell 1994, S. 11.

12 Vgl. Robinson, Howard: »Dualism«, in: The Stanford Encyclopedia of Philosophy, 2013, Online: http://plato.stanford.edu/archives/fall2013/entries/dualism/ [zuletzt geprüft am 25.09.2018], Abschnitt 2.1.

13 Genauer: der Eigenschaftsdualismus geht im Gegensatz zum Prädikatendualismus davon aus, dass die Ontologie der Physik nicht hinreichend für die existierenden Phänomene ist: »Genuine property dualism occurs when, even at the individual level, the ontology of physics is not sufficient to constitute what is there «. A.a.O., Abschnitt 22 [Hervorhebung E. E.]. Aus diesem Grunde sind für Eigenschaftsdualisten Qualia nichtphysikalische Eigenschaften. Vgl. Feser, Edward: »Qualia. Irreducibly Subjective but not Intrinsic«, Journal of Consciousness Studies, [2001] Vol. 8, No. 8, S. 3-4.

14 Da der Dual-Aspekt-Monismus von der Irreduzibilität von Geist und Materie ausgeht, kann man bei ihm noch von einer subtilen Form des Dualismus sprechen.

15 Atmanspacher, Harald: »Dual-Aspect Monism à la Pauli and Jung«, Journal of Consciousness Studies, [2012] Vol. 19, No. 9-10, S. 97.

16 Vgl. ebenfalls Atmanspacher, Harald: »Quantum Approaches to Consciousness «, in: The Stanford Encyclopedia of Philosophy, 2015, Online: http://plato.stanford.edu/archives/ sum2015/entries/qt-consciousness [zuletzt geprüft am 28.o9.2018], Abschnitt 4.6.

17 Als früher Vertreter des neutralen Monismus kann Spinoza gesehen werden, vgl. dazu: Seager, William / Allen-Hermanson, Sean: »Panpsychism«, in: The Stanford Encyclopedia of Philosophy, 2013, Online: http://plato.stanford.edu/archives/fall2013/ entries/panpsychism/ [zuletzt geprüft am 25.09.2018], Abschnitt 3.o. Ebenfalls als Vorreiter des neutralen Monismus gelten z.B. Ernst Mach, William James, and Bertrand Russell. Vgl. Atmanspacher, Harald: »Dual-Aspect Monism à la Pauli and Jung «,Journal of Consciousness Studies, [2012] Vol. 19, No. 9-10, S. 98.

18 Vgl. Velmans, Max: »Reflexive Monism. Psychophysical Relations among Mind, Matter, and Consciousness«, Journal of Consciousness Studies, [2012] Vol. 19, No. 9-10, S. 144. 
mentale Eigenschaften auf ontologisch »neutralen «19 Eigenschaften gründen. Diese neutrale Eigenschaft ist weder als >physikalisch $<$ noch >mental zu verstehen. Solche Formen des neutralen Monismus können deshalb als Eigenschaftsmonismen verstanden werden, die jedoch nicht reduktiv-physikalistisch sind. Denn nach diesen Positionen gibt es, ontologisch gesehen, weder unabhängige physikalische noch unabhängige mentale Eigenschaften. ${ }^{20}$

Auf der monistischen Seite dieser Achse sind nicht nur reduktionistischphysikalistische Positionen mit der Ontologie eines materialistischen Monismus, zu verorten. Ebenfalls eine strikt monistische Ontologie haben Positionen mit einem immaterialistischen Monismus, wie z.B. die (immaterialistisch-) monistische Variante des Panpsychismus, ${ }^{21}$ der Solipsismus oder Varianten des Idealismus, nach welchen der Geist die letzte Grundlage der Realität ist. ${ }^{22}$ Die strikteste Form eines materialistisch-reduktionistischen Monismus lässt sich wohl in einem Prädikatenmonismus sehen, nach welchem nur schon die Rede von Geisteszuständen auf einem >Irrtum < beruht. Denn wenn Geisteszustände nicht >existieren<, weil sie »nichts anderes als« (»nothing other than«) ${ }^{23} \mathrm{Ge}-$ hirnzustände sind, dann reichen physikalische Prädikate vollständig aus, um Geisteszustände zu beschreiben. ${ }^{24}$

19 Alter, Torin / Nagasawa, Yujin: »What is Russellian Monism?«, Journal of Consciousness Studies, [2012] Vol. 19, No. 9-10, S. 78.

20 Vgl. ebd.

21 Zur immaterialistisch-monistischen Variante des Panpsychismus vgl. Clifford, William: »On the Nature of Things-in-Themselves«, Mind, [1878] Vol. 3, No. 9, S. 57-67. Der Panpsychismus kann jedoch auch in einer Dual-Aspekt-Version oder in Varianten des neutralen Monismus vorkommen. Vgl. Weekes, Anderson: »The Mind-Body Problem and Whitehead's Non-reductive Monism «, Journal of Consciousness Studies, [2012] Vol. 19, No. 9-10, S. 49. Bezüglich der Variante des neutralen Monismus vgl. Seager, William / Allen-Hermanson, Sean: »Panpsychism«, in: The Stanford Encyclopedia of Philosophy, 2013, Online: http://plato.stanford.edu/archives/fall2o13/entries/panpsychism/ [zuletzt geprüft am 25.09.2018], Abschnitt 3.0.

22 Vgl. dazu Guyer, Paul / Horstmann, Rolf-Peter: »Idealism «, in: The Stanford Encyclopedia of Philosophy, 2015, Online: http://plato.stanford.edu/archives/fall2015/entries/idealism/ [zuletzt geprüft am 26.o9.2016], Abschnitt 1.o.

23 So konstatiert Dennett: »I claim, then, that sensory qualities the dispositional properties of cerebral states to produce certain further effects in the very observers whose states they are«. Dennett, Daniel C.: »Lovely and Suspect Qualities«, 1991, Online: http://cogprints. org/263/1/lovely\%26s.htm [zuletzt geprüft am 24.09.2018]. Zur Kritik der >nicht anders als<-These, vgl auch Searle, John R.: The mystery of consciousness, New York: New York Review Books 1997, S. 210. Searle kritisiert diesbezüglich treffend: »They want to claim that it is >nothing but ...<- and then they pick their favourite candidate to fill the blank $[\ldots] \ll$. Ebd.

24 Vgl. Dennett, Daniel C.: »Quining Qualia«, in: Philosophy of mind. Classical and contemporary readings, Chalmers, David J. [Hrsg.], New York: Oxford University Press [zuerst 1988]/2002, S. 227 . 
Betrachten wir das hard problem durch die Problemoptik der ontologischen Achse, fällt auf, dass nicht nur substanzdualistische, sondern auch strikt monistische Konzeptionen, sich auf einen als >evident erachteten Grundsatz (bzw. auf die Nicht-Akzeptanz des Grundsatzes der Gegenseite) berufen. ${ }^{25}$ Interessant ist auch die Parallele, im Hinblick auf die ontologische Sparsamkeit zwischen den strikten Formen von Monismen wie beispielsweise eliminativistisch-materialistischen und solipsistischen immaterialistischen Monismen. Diesen Formen äusserster ontologischer Sparsamkeit könnte ein intensiver Wunsch nach ontologischer Einfachheit zu Grunde liegen. Was die dogmatisch anmutenden Behauptungen und zuweilen zirkulären Argumentationsstrukturen auf denen sowohl Solipsismus als auch Eliminativismus beruhen, erklären könnte (vgl. Abschnitte 4.2.1 und 6.5).

\subsection{Mereologische Achse: Mereologischer Reduktionismus vs. mereologischer Holismus}

Auf der Achse dieser Problemoptik stehen sich gewissermassen »holistische « ${ }^{26}$ Konzeptionen und »mereologische Reduktionismen «27 von bewusstem Erleben gegenüber. Auf der Seite des mereologischen Reduktionismus gelten die Eigenschaften der (neuronalen) Bestandteile von Erleben als »kontextunabhängig «, ${ }^{28}$ weshalb man diesbezüglich auch von identitätstheoretischen $>$ Lokalisierungen< von mentalen Zuständen sprechen könnte. So geht man in stärkeren Formen des Repräsentationalismus davon aus, dass Qualia identisch mit bestimmten (nicht-begrifflichen) Formen repräsentationaler Gehalte sind. ${ }^{29}$ Bei solchen Formen des Repräsentationalismus spricht man

25 Vgl. dazu auch Schildknecht, Christiane: »Der Dualismus und die Rettung der Phänomene «, in: Homo Sapiens und Homo Faber. Epistemische und technische Rationalität in Antike und Gegenwart. Festschrift für Jürgen Mittelstrass, Wolters, Gereon / Mittelstrass, Jürgen [Hrsg.], Berlin: W. de Gruyter 2005, S. 225. Zentral für die Argumentation beider Argumentationslinien ist nach Schildknecht die der Beweislastverschiebung. Vgl. ebd.

26 Die Konzeption eines >Holismus des Mentalen<, geht mindestens von der Irreduzibilität von mentalen Begriffen (»the irreducibility of mental concepts«) aus. Davidson, Donald: »Davidson, Donald«, in: A companion to the philosophy of mind, Guttenplan, Samuel [Hrsg.], Malden, Massachusetts: Blackwell 2005, S. 231.

27 Weekes, Anderson: »The Mind-Body Problem and Whitehead's Non-reductive Monism «, Journal of Consciousness Studies, [2012] Vol. 19, No. 9-10, S. 55.

28 Ebd.

29 Tye, Michael: »Qualia«, in: The Stanford Encyclopedia of Philosophy, 2007, Online: http:// plato.stanford.edu/archives/fall2007/entries/qualia/ [zuletzt geprüft am 25.09.2018], Abschnitt 7.o. Vgl. dazu auch Pitt, David: »Mental Representation«, in: The Stanford Encyclopedia of Philosophy, 2008, Online: http://plato.stanford.edu/archives/fall2008/ entries/mental-representation [zuletzt geprüft am 25.09.2018], Abschnitt 4.o. 
von >engen< bzw. sinternen $<$ Gehalten ${ }^{30}$ - den Hirnzuständen -, die identisch mit Qualia seien. ${ }^{31}$ Ebenfalls setzen Identitätstheorien auf eine strikte psychophysische Identität von bestimmten Hirnzuständen und Bewusstsein. ${ }^{32}$ Ein Erlebnis zu haben »[...] just is being in a brain state with the relevant neurophysiological properties [... « ${ }^{33}$ Gemeinsam ist den Zugängen auf dieser Seite der mereologischen Achse demnach, dass davon ausgegangen wird, dass das >Hirn< sieht, denkt, hört. Anders gesagt: alles Erleben ist »[...] eine Konvention des Gehirns mit sich selbst «. ${ }^{34}$

Holistische Ansätze gehen generell davon aus, dass die Eigenschaften der Bestandteile eines Systems mindestens teilweise vom ganzen System, mit dem sie zusammenhängen, bestimmt sind. ${ }^{35}$ Konzeptionen mentaler Repräsentation, die neben der Umwelt auch die ganze Person mit ihren Wünschen und Zielen einbeziehen, befinden sich auf der mereologischen Achse der Problemoptik demzufolge mehr auf der Seite >holistischer Zugänge zum Phänomenalen. ${ }^{36}$ Je nach Ausprägung, können hierzu schwächere Formen des Repräsentationalismus oder nicht-reduktive Formen des Funktionalismus

30 Demgegenüber werden in externalistischen Varianten des Repräsentationalismus (»wide content«) Qualia als geistunabhängige, objektive Eigenschaften von Gegenständen gesehen. Vgl. Dretske, Fred: »Woher wissen Sie, dass Sie kein Zombie sind? «, in: Den eigenen Geist kennen. Selbstwissen, privilegierter Zugang und Autorität der ersten Person, Newen, Albert / Vosgerau, Gottfried [Hrsg.], Paderborn: Mentis [zuerst 2003, engl.]/2005, S. 102 und 109.

31 Vgl. Staudacher, Alexander: Phänomenales Bewusstsein als Problem für den Materialismus, Berlin: De Gruyter 2002, S. 329. Vgl. ebenfalls Lycan, William: »Representational Theories of Consciousness «, in: The Stanford Encyclopedia of Philosophy, 2008, Online: http://plato. stanford.edu/archives/fall2008/entries/consciousness-representational/ [zuletzt geprüft am 28.09.2018], Abschnitt 2.2.

32 Vgl. Papineau, David:»Mind the Gap«, Philosophical Perspectives, [1998] Vol. 12: Language, Mind, and Ontology, S. 379. Vgl. ebenfalls Van Gulick, Robert: »Consciousness«, in: The Stanford Encyclopedia of Philosophy, 2014, Online: http://plato.stanford.edu/archives/ spr2014/entries/consciousness/ [zuletzt geprüft am 17.09.2018], Abschnitt 8.2.

33 Ebd. [Hervorhebung im Original].

34 Roth, Gerhard: Das Gehirn und seine Wirklichkeit. Kognitive Neurobiologie und ihre philosophischen Konsequenzen, Frankfurt am Main: Suhrkamp 1994, S. 328 [Hervorhebung im Original].

35 Vgl. Weekes, Anderson: »The Mind-Body Problem and Whitehead's Non-reductive Monism«, Journal of Consciousness Studies, [2012] Vol. 19, No. 9-10, S. 55.

36 In diesen Zugängen geht es nicht zwingend um eine Ontologie mentaler Zustände, jedoch oft um ein umfassenderes Verständnis des Menschen als Subjekt und als Objekt lebensweltlicher Zusammenhänge. Vgl. dazu auch Marienberg, Sabine: »Die Grenzen der Vergegenständlichung qualitativen Erlebens«, in: Funktionen des Erlebens. Neue Perspektiven des qualitativen Bewusstseins. Band 5: Humanprojekt, Jung, Matthias / Heilinger, JanChristoph [Hrsg.], Berlin: De Gruyter 2009, S. 43. 
gezählt werden. ${ }^{37}$ Schwächere Formen des Repräsentationalismus wie z.B. die von Block, Chalmers, Loar und Peacocke gehen zwar auch davon aus, dass Qualia (funktional) repräsentiert sind. Im Gegensatz zu den Theorien des starken Repräsentationalismus, die davon ausgehen, dass der phänomenale Charakter eines mentalen Zustandes auf intentionalen Gehalt zu reduzieren sei, gehen schwache Repräsentationalisten davon aus, dass der phänomenale Charakter irreduzibel ist. ${ }^{38}$ Für schwache Repräsentationalisten muss deshalb ein mentaler Zustand nicht zwingend mit dem Repräsentat (bzw. einem Hirnzustand $)^{39}$ identisch und somit auch nicht >identitätstheoretisch lokalisiert< sein.

Bennett und Hacker kritisieren die Redeweise vom Gehirn, welches >denken<, >fühlen< oder >entscheiden< soll. Eine dergestaltige Zuschreibung von psychologischen Attributen des Hirns lässt sich nach ihnen als »mereologischen Trugschluss« (»mereological fallacy« $)^{40}$ diagnostizieren, der sich neurowissenschaftlich gar nicht stützen lässt. ${ }^{41}$ Die Rede vom >Gehirn<, das etwas sempfindet<, gründet vielmehr in einer begrifflichen Fehlanwendung, ${ }^{42}$ da

37 Vgl. dazu Pitt, David: »Mental Representation«, in: The Stanford Encyclopedia of Philosophy, 2008, Online: http://plato.stanford.edu/archives/fall20o8/entries/mentalrepresentation [zuletzt geprüft am 25.09.2018], Abschnitt 4.o.

38 Vgl. dazu ebd. Vgl. z.B. auch Block, Ned J.: »Inverted Earth«, in: Philosophy of mind. Critical concepts in philosophy. Volume 4: Consciousness, Crawford, Sean [Hrsg.], London: Routledge \& Kegan Paul [zuerst 199o]/2011, S. 121. Vgl. auch Block, Ned J.: »Mental Paint and Mental Latex«, Philosophical Issues, [1996] Vol. 7: Perception, S. 19-49. Vgl. ebenfalls Chalmers, David J.: The conscious mind. In search of a fundamental theory, New York: Oxford University Press 1996, S. 249.

39 Das Repräsentat (nicht zu verwechseln mit Repräsentant) ist der Träger einer Repräsentation, wie z.B. ein Gehirnzustand. Nach Metzinger ist mentale Repräsentation eine dreistellige Repräsentation, die aus Repräsentand (Objekt), Repräsentat (Träger der Repräsentation, Gehirnzustand) und System (ganze Person mit Wünschen und Zielen) besteht. Demgemäss gesteht Metzinger auch ein, dass das Gehirn als solches nichts fühlt und auch nichts denkt. Vgl. Metzinger, Thomas [Hrsg.]: Grundkurs Philosophie des Geistes. Band 3: Intentionalität und mentale Repräsentation, Paderborn: Mentis 2007, S. 24. Vgl. auch Metzinger, Thomas [Hrsg.]: Grundkurs Philosophie des Geistes. Band 2: Das LeibSeele-Problem, Paderborn: Mentis 2007, S. 220.

40 Bennett, Maxwell R. / Hacker, Peter M.: Philosophical foundations of neuroscience, Malden, Massachusetts: Blackwell 2003, S. 68.

41 »The ascription of psychological attributes to the brain is not warranted by a neuroscientific discovery [...] «. A.a.O., S. 72.

42 So stellen Bennett und Hacker fest: »The brain is not a logically appropriate subject for psychological predicates«. Ebd. Für eine detailliertere sprachkritische Auseinandersetzung mit problematischen Begriffsverwendungen in der Neurowissenschaft, vgl. Janich, Peter: Kein neues Menschenbild. Zur Sprache der Hirnforschung, Frankfurt am Main: Suhrkamp 20o9. 
physikalische und psychologische Beschreibungsebenen inkompatibel sind.43 Wir können eine Person mitsamt ihrem Verhalten beobachten und (mit einer gewissen Fehlerquote) feststellen, ob sie einen Schmerz fühlt oder einen Gegenstand sieht. Können wir aber behaupten, dass ihr Hirn etwas >glaubt< oder etwas $>$ sieht $?^{44}$ Eine mereologisch kritische Frage gegenüber solchen Konzeptionen lautet daher: »[...] do we know what it is for a brain to see or hear, for a brain to have experiences, to know or believe something? [...] Do we grasp what it is for a brain (let alone a neuron) to reason $[\ldots .]. ?{ }^{45}$ Eine holistische Kritik am mereologischen Fehlschluss finden wir bei Fuchs, dessen Aufsatztitel Hirnwelt oder Lebenswelt? Zur Kritik des Neurokonstruktivismus die Polarität dieser Problemachse schon treffend umschreibt: »[...] es ist nicht das Gehirn, das fühlt, denkt, wahrnimmt oder sich bewegt, sondern nur das Lebewesen, der lebendige Organismus als ganzer «. ${ }^{46}$ Mereologisch kritisch ist auch Damasio. Für ihn ist eine Konzeption des Geistes im Sinne eines isolierten Gehirns beinahe undenkbar: »The near inevitability of body processing, regardless of what it is that we are doing or thinking, should be apparent. Mind is probably not conceivable without some sort of embodiment $[\ldots] \ll{ }^{47}$ Mentales beschränkt sich nicht einfach auf einen Prozess >im Gehirn<, sondern betrifft den ganzen Organismus: »Making mind arise out of an organism rather than out of a disembodied brain $[\ldots] \ll{ }^{48}$ Körperliche Prozesse sind für den Geist eine unabdingbare Basis: »[...] the body provides a ground reference for the mind « ${ }^{49}$ So bemerkt Damasio, dass sich hinter dem Denken von Neurowissenschaftern, die behaupten, dass der Geist vollständig mit »Hirnvorgängen « ${ }^{50}$ erklärt werden könne, die Idee einer »Cartesianischen Entkörperung « (»Cartesian disembodiment« $)^{51}$ verbergen könnte. Hermann

43 Vgl. dazu auch Metzinger, Thomas [Hrsg.]: Grundkurs Philosophie des Geistes. Band 2: Das Leib-Seele-Problem, Paderborn: Mentis 2007, S. 220.

44 Mit anderen Worten: Die Zuschreibung der Tätigkeit des Sehens auf ein >Gehirn $<$ - statt auf eine Person - angewendet, scheint eher unsinnig: »[...] what would it be to observe whether a brain sees something - as opposed to observing the brain of a person who sees something [...]?«. Bennett, Maxwell R. / Hacker, Peter M.: Philosophical foundations of neuroscience, Malden, Massachusetts: Blackwell 2003, S. 70 [Hervorhebung E.E.].

45 Ebd. [Hervorhebung im Original]. Vgl. dazu auch Krüger, Hans-Peter [Hrsg.]: Hirn als Subjekt? Philosophische Grenzfragen der Neurobiologie, Berlin: Akademie-Verlag 2009.

46 Fuchs, Thomas: »Hirnwelt oder Lebenswelt? Zur Kritik des Neurokonstruktivismus«, Deutsche Zeitschrift für Philosophie, [2011] Vol. 59, No. 3, S. 356.

47 Damasio, Antonio R.: Descartes' error. Emotion, reason and the human brain, London: Vintage [zuerst 1994]/2006, S. 234.

48 A.a.O., S. 229.

49 A.a.O., S. 223.

50 A.a.O., S. 251.

51 A.a.O., S. 25 o. 
Schmitz spricht in diesem Zusammenhang sogar von einer »Gehirnmythologie «,52 die sich selbst aufhebe. Nach ihm ist die Rede über das Gehirn, die »Achillesferse $\aleph^{53}$ materialistischer Positionen der Philosophie des Geistes. Mit mereologischem Problembewusstsein konstatiert schliesslich Stubenberg, dass sobald unser vorphilosophischer, naiver Realismus seine ersten Hiebe ausgeteilt habe, wir danach weder an physikalischen Objekten noch in einem $>$ Hirn`Qualia ausmachen können. ${ }^{54}$

\subsection{Modale Achse: Supervenienz vs. Kontingenz}

Betrachten wir das hard problem durch die Problemoptik, welche ich als $>$ modale Achse $<$ bezeichne, finden wir, grob gesprochen, folgende zwei gegensätzliche Konzeptionsvarianten über Bewusstseins: Entweder geht man von einer Supervenienz oder aber von einer Kontingenz von Erleben (im Zusammenhang mit damit korrelierenden physikalischen Zuständen) aus. Eine zentrale Intuition materialistischer Zugänge zum hard problem besteht darin, dass die Beziehung zwischen dem Mentalen und dem Physischen eine asymmetrische Beziehung sein müsse. Das heisst, dass bestimmte Phänomene »fundamentaler « ${ }^{55}$ als andere seien und deshalb das Mentale über dem Physischen zwingend superveniere. Die stärkste Ausprägung auf der Seite solcher Supervenienzthesen, haben Konzeptionen des Mentalen, die davon ausgehen, dass Bewusstsein logisch zwingend über der Supervenienzbasis physikalischer bzw. neuronaler Zustände superveniert. Wird davon ausgegangen, dass dies nur metaphysisch zwingend ${ }^{56}$ ist, gesteht man mindestens ein, dass Bewusstsein nicht logisch zwingend über physikalischen Zuständen supervenieren muss. Noch etwas weniger auf der Seite der Supervenienzthesen, sind Ansätze, die einräumen, dass es weder logisch noch metaphysisch

$5^{2}$ Schmitz, Hermann / Sohst, Wolfgang: Hermann Schmitz im Dialog. Neun neugierige und kritische Fragen an die Neue Phänomenologie, Berlin: Xenomos 2005, S. 48.

53 Ebd.

54 Stubenberg, Leopold: Consciousness and qualia, Amsterdam: John Benjamins 1998, S. 26. Ähnlich argumentiert Nagel, vgl. Nagel, Thomas: Der Blick von nirgendwo, Frankfurt am Main: Suhrkamp [zuerst 1986, engl.]/1992, S. 8 o und 84.

55 Staudacher, Alexander: Phänomenales Bewusstsein als Problem für den Materialismus, Berlin: De Gruyter 2002, S. 16.

$5^{6}$ Beim Spielraum metaphysischer Möglichkeit geht es nicht nur um den Spielraum des Denkbaren, sondern darum, was in einer möglichen Welt tatsächlich der Fall sein könnte, und was nicht bzw. was metaphysisch zwingend ist. 
zwingend, sondern nur nomologisch zwingend ${ }^{57}$ ist, dass Bewusstsein über physikalischen Zuständen superveniert. ${ }^{58}$

Auf der Seite der Kontingenzthesen des Bewusstseins, finden sich Konzeptionen, die allgemein davon ausgehen, dass Bewusstsein weder aus physikalischen Tatsachen abgeleitet werden kann, noch dass es mit Physikalischem identisch sein muss. ${ }^{59}$ Demnach finden sich einerseits Theorien des Geistes, die sich mit modalen Argumenten über die Möglichkeit von $>$ Zombies ${ }^{60}$ gegen Identitätstheorien wenden und andererseits explanatorische Argumente, die sich auf einen »sense of arbitrariness « 61 zwischen neuronalem Zustand und einem Erlebnis berufen. ${ }^{62}$ Die stärksten Varianten von Kontingenzthesen gehen dementsprechend davon aus, dass es weder logisch, noch metaphysisch, noch nomologisch zwingend ist, dass Bewusstsein über physikalischen oder neuronalen Zuständen superveniert. ${ }^{63}$

$57 \quad$ Nomologisch zwingend ist alles, was unter Berücksichtigung der physikalischen Gesetze zwingend ist. Dieser Möglichkeitsrahmen gilt als enger, als der Rahmen des logisch Zwingenden und, je nach Definition metaphysischer Möglichkeit, wird ein nomologischer Möglichkeitsrahmen enger als derjenige metaphysischer Modalität verstanden. Vgl. dazu auch Chalmers, David J.: The conscious mind. In search of a fundamental theory, New York: Oxford University Press 1996, S. 134. Vgl. ebenfalls Staudacher, Alexander: Phänomenales Bewusstsein als Problem für den Materialismus, Berlin: De Gruyter 2002, S. 397.

58 Mehr zu diesem Punkt findet sich bereits im Abschnitt über modale Argumente (4.1.3).

59 Vgl. z.B. Chalmers, David J.: The conscious mind. In search of a fundamental theory, New York: Oxford University Press 1996, S. 140.

6 o Vgl. a.a.O., S. 94-99.

61 Levine, Joseph: »Phenomenal concepts and the materialist constraint «, in: Phenomenal concepts and phenomenal knowledge. New essays on consciousness and physicalism, Alter, Torin / Walter, Sven [Hrsg.], Oxford: Oxford University Press 2007, S. 145.

62 Vgl. dazu Levine, Joseph: »Materialism and Qualia: The Explanatory Gap«, Pacific Philosophical Quarterly, [1983] Vol. 64, No. 4, S. 356. Vgl. auch Levine, Joseph:»Phenomenal concepts and the materialist constraint «, in: Phenomenal concepts and phenomenal knowledge. New essays on consciousness and physicalism, Alter, Torin / Walter, Sven [Hrsg.], Oxford: Oxford University Press 2007, S. 145.

63 Ein Beispiel für diese Konzeptionsvariante minimaler bzw. nicht vorhandener Supervenienz von Bewusstsein über Physikalischem wären m.E. der klassische, substanzdualistischen Cartesianismus oder zeitgenössische Formen des Substanzdualismus, wie z.B. denjenigen von Swinburne oder Foster. Vgl. dazu Swinburne, Richard: »Body and Soul«, in: The mind-body problem. A guide to the current debate, Warner, Richard / Szubka, Tadeusz [Hrsg.], Cambridge, Massachusetts: Blackwell 1994, S. 311-316. Vgl. auch Foster, John: The immaterial self. A defence of the Cartesian dualist conception of the mind, London: Routledge \& Kegan Paul 2005. 
Achse kausaltheoretischer Überlegungen: Epiphänomenalismus vs. kausale Relevanz des Mentalen

Auf dieser Achse befinden sich einerseits epiphänomenalistische Theorien des Geistes und andererseits Konzeptionen, die dem Mentalen eine kausale Relevanz zugestehen. Epiphänomenalistische Konzeptionen basieren auf der Überlegung, dass aufgrund des Prinzips der kausalen Geschlossenheit unserer Welt Mentales (vermeintlich) ${ }^{64}$ nur dann existieren kann, wenn es ein Epiphänomen, d.h. kausal irrelevant ist. Mit dieser These versucht der Epiphänomenalist, zwei konfligierende Überzeugungen zu vereinen: a.) moderne Naturwissenschaft lässt nur Kausalitäten zu; b.) das Erleben von mentalen Zuständen ist nicht vollständig mit neuronalen Prozessen identifizierbar. ${ }^{65}$ Die stärkste Spielart des Epiphänomenalismus finden wir im klassischen Epiphänomenalismus von Huxley. Nach diesem ist Bewusstsein in kausaler Hinsicht mit der Dampf-Pfeife einer Lokomotive vergleichbar: der Dampfmaschinenmechanismus bewirkt zwar das Pfeifen, letzteres wirkt aber nicht auf die Dampfmaschine zurück und ist insgesamt irrelevant für den Dampfmechanismus. ${ }^{66}$ So scheint es nach dem Epiphänomenalismus nur so, als ob unser Verhalten von Überzeugungen oder Empfindungen verursacht sei. Kausal relevant sind nur physikalische Prozesse im Gehirn und im Nervensystem, nicht aber das Erleben der mentalen Zustände bzw. Bewusstsein, das folglich als blosses Epiphänomen gilt. ${ }^{67}$ Aufgrund der postulierten kausalen Irrelevanz von bewusstem Erleben wird geschlossen, dass Erklärungsmodelle für kognitive Leistungen des Gehirns völlig von der Erlebniskomponente absehen sollen. ${ }^{68}$ Schwächere Formen des Epiphänomenalismus gehen hingegen davon aus, dass nur einige Eigenschaften von mentalen Zuständen kausal

64 Vgl. dazu weiter unten.

65 Ebenfalls könnte der Epiphänomenalismus dafür dienen, den gefürchteten interaktionistischen Dualismus, der aus den divergierenden Überzeugungen a.) und b.) folgen könnte, zu vermeiden. Vgl. dazu auch Walter, Sven: »Epiphenomenalism«, in: The Oxford Handbook of Philosophy of Mind, McLaughlin, Brian P. / Beckermann, Ansgar / Walter, Sven [Hrsg.], Oxford: Oxford University Press 2009, S. 86.

66 Vgl. dazu Huxley, Thomas H.: »On the hypothesis that animals are automata, and its history«, in: Collected Essays, Volume I, Method and Results, Huxley, Thomas H. [Hrsg.], London: Macmillan [zuerst 1874]/1893, S. 240-244.

67 Vgl. dazu Walter, Sven: »Epiphenomenalism«, in: The Oxford Handbook of Philosophy of Mind, McLaughlin, Brian P. / Beckermann, Ansgar / Walter, Sven [Hrsg.], Oxford: Oxford University Press 2009, S. 86.

68 Roth kritisiert diese These, vgl. weiter unten. Vgl. ebenfalls Roth, Gerhard: Das Gehirn und seine Wirklichkeit. Kognitive Neurobiologie und ihre philosophischen Konsequenzen, Frankfurt am Main: Suhrkamp 1994, S. 294-295. 
irrelevant sind, während eine dritte Spielart des Epiphänomenalismus davon ausgeht, dass nur Qualia epiphänomenal sind. ${ }^{69}$ In letzterer Spielart können zwar Qualia als kausal irrelevant, die intentionalen Eigenschaften von propositionalen Einstellungen jedoch als kausal relevant gelten. ${ }^{70}$

Der anomale Monismus von Davidson ${ }^{71}$ nimmt auf dieser Achse eine Sonderstellung ein, indem er davon ausgeht, dass es keine strikten psychophysischen Gesetze gibt, die es ermöglichen, menschliche Gedanken und Handlungen vorauszusagen. Demzufolge sind psychologische Kausalitäten nicht auf physikalische Kausalitäten reduzierbar, obschon für den anomalen Monismus mentale Zustände identisch mit physikalischen Zuständen sind. Die Identität gilt jedoch nur im Hinblick auf partikulare mentale und neuronale Zustände, d.h. im Hinblick auf so genannte »tokens « ${ }^{72}$ Und weil es nach dem anomalen Monismus keine von herkömmlichen Identitätstheorien postulierten Typenidentität gibt, kann es auch keine strikte gesetzesartige Kausalität zwischen mentalen und physischen Eigenschaften geben. ${ }^{73}$

Für Konzeptionen, die von einer kausalen Relevanz des Mentalen ausgehen, lässt sich - gegen den Epiphänomenalismus - einwenden, dass es offenbar nicht möglich ist, Bewusstsein von bestimmten funktionalen bzw. kognitiven Leistungen abzutrennen. Wenn wir bspw. bei komplizierten Aussagen nicht bewusst hinhören, entgeht uns typischerweise ihr Sinn. Ähnliches gilt für das Erlernen komplizierter motorischer Fertigkeiten oder beim Lesen. ${ }^{74}$ Weil Beeinträchtigungen des subjektiven Bewusstseinszustandes mit einer Verminderung bestimmter kognitiver Leistungen einhergehen, gehen deshalb Kritiker des Epiphänomenalismus davon aus, dass Bewusstsein kausal relevant sein muss. ${ }^{75}$ Weitere Einwände gegen den Epiphänomenalismus bestehen

69 Vgl. dazu auch Chalmers, der feststellt, dass seine Theorie des Geistes mindestens eine schwache Form des Epiphänomenalismus impliziere: Chalmers, David J.: The conscious mind. In search of a fundamental theory, New York: Oxford University Press 1996, S. 16o.

70 Vgl. Staudacher, Alexander: Phänomenales Bewusstsein als Problem für den Materialismus, Berlin: De Gruyter 2002, S. 427.

71 Vgl. Davidson, Donald: »Mental Events«, in: Essays on actions and events, Davidson, Donald [Hrsg.], Oxford: Clarendon Press [zuerst 1970]/2002, S. 207-224.

72 Yalowitz, Steven: »Anomalous Monism«, in: The Stanford Encyclopedia of Philosophy, 2012, Online: https://plato.stanford.edu/entries/anomalous-monism/ [zuletzt geprüft am 25.09.2018], Abschnitt einleitender Erläuterungen.

73 Vgl. ebd.

74 Vgl. Roth, Gerhard: Das Gehirn und seine Wirklichkeit. Kognitive Neurobiologie und ihre philosophischen Konsequenzen, Frankfurt am Main: Suhrkamp 1994, S. 294.

Vgl. ebd. 
im Verweis auf evolutionstheoretische Argumente, ${ }^{76}$ auf die Beziehung zwischen unserem bewussten Erleben und unseren Handlungen ${ }^{77}$ oder auf die Verbindung zwischen bewusstem Erleben und unseren Urteilen ${ }^{78}$ über das Erlebte. Der Einfluss, den bewusstes Erleben auf Urteile hat, kann auch im $\mathrm{Zu}-$ sammenhang mit der Entstehung von Theorien als Einwand gegen den Epiphänomenalismus geltend gemacht werden. ${ }^{79}$ Positionen, die für eine kausale Relevanz von Bewusstsein plädieren, erheben auch systematische Einwände, die darin bestehen, dass Erlebnisse, wenn sie gleichsam als »nomologisch frei schwebende Entitäten« (»nomological danglers«) $)^{80}$ bestünden, höchst unplausible Eigenschaften dieser Welt wären.

Theorien des Geistes, welche Bewusstsein nicht im Sinne eines materialistischen Monismus ontologisch reduzieren, müssen sich aber gar nicht auf

76 Wenn Bewusstsein ein Produkt der Evolution und natürlicher Selektion sind, dann muss es auch eine Wirkung auf die Anpassungsfähigkeit eines Organismus gehabt haben. Dem widerspricht jedoch die Prämisse der kausalen Irrelevanz des Phänomenalen. Vgl. dazu Walter, Sven: »Epiphenomenalism«, in: The Oxford Handbook of Philosophy of Mind, McLaughlin, Brian P. / Beckermann, Ansgar / Walter, Sven [Hrsg.], Oxford: Oxford University Press 2009, S. 87.

77 Beispiel: Aufgrund des Erlebens von Schmerzen greifen wir zu Schmerztabletten. Oder beim Verhalten einer anderen Person, die offenbar einen Wutanfall hat, wird die von ihr erlebte Emotion als Auslöser für ihren Wutanfall gesehen. Für einen Epiphänomenalisten ist diese Erklärung jedoch nicht möglich, da nach ihm die Emotion keinen kausalen Einfluss auf das Verhalten haben kann. Vgl. dazu ebd.

78 Beispiel: Die Aussage sich habe Schmerzen< liesse sich für einen Epiphänomenalisten nicht so interpretieren, dass sie durch das Erleben des Schmerzes (mit-) verursacht wurde. Vgl. dazu Schröder, Jürgen: »Qualia und Physikalismus«, Journal for General Philosophy of Science, [1997] Vol. 28, No. 1, S. 167.

79 Denn es lässt sich feststellen, dass Erleben als (vermeintliches) Epiphänomen, mindestens insofern kausal relevant sein muss, dass aufgrund dieser Erlebniskomponente eine Theorie, wie die des Epiphänomenalismus überhaupt ausgedacht wird. Anders gesagt: es käme wohl niemand auf eine epiphänomenalistische Theorie des Geistes, wenn es nicht das Explanandum mentaler Zustände gäbe, die man mit dieser Theorie in eine kausal geschlossene Welt integrieren möchte. (Mit dem Epiphänomenalismus soll ja das Problem gelöst werden, wie das Erleben mentaler Zustände in eine kausal geschlossene Welt passt.) Dann aber ist das Erleben von mentalen Zuständen mindestens insofern kausal relevant, dass aufgrund dieser, wie auch immer gearteten Erscheinung, epiphänomenalistische Theorien des Geistes ausgedacht worden sind. Infolgedessen ist aber das Erleben mentaler Zustände die kausale Ursache dafür, dass es den Epiphänomenalismus gibt. Daraus folgt, dass phänomenale oder zumindest bewusste mentale Zustände, entgegen dem Epiphänomenalismus, kausal relevant sind. Der Epiphänomenalismus könnte dagegen vielleicht noch folgendes, ziemlich seltsames Gegenargument einbringen: unser Erleben ist auch irrelevant im Hinblick auf die (epiphänomenalistische) Erklärung, warum wir überhaupt von Erleben sprechen. Vgl. dazu Chalmers, David J.: The conscious mind. In search of a fundamental theory, New York: Oxford University Press 1996, S. 159. 
den Epiphänomenalismus berufen; gleichzeitig müssen sie nicht zwingend in Konflikt mit der Prämisse der kausalen Geschlossenheit der physikalischen Welt geraten. Theorien, wie der neutrale Monismus, Panpsychismus oder der nicht-reduktive Funktionalismus können bewusstem Erleben kausale Relevanz zusprechen ohne es auf physikalische Prozesse zu reduzieren. So weist bspw. Atmanspacher darauf hin, dass mentale und materielle Eigenschaften miteinander korrelieren können, weil sie zusammen auf einer neutralen, psychophysischen Ebene verursacht wurden. Folglich gibt es nach diesem Ansatz nur keine direkten kausalen Interaktionen zwischen mentalen und materiellen Eigenschaften, obwohl sie miteinander korrelieren. ${ }^{81}$

\subsection{Achse explanatorisch-reduktionstheoretischer und epistemischer Optiken}

Im Hinblick auf ihre Problemoptik haben die epistemische, die explanatorische und die reduktionstheoretische Achse sehr viele Überlappungen. Deshalb werde ich diese drei Achsen im folgenden Abschnitt gemeinsam behandeln.

Epistemisch bzw. explanatorisch optimistische Konzeptionen des hard problem gehen typischerweise davon aus, dass Dritte-Person-Zugänge zum Bewusstsein möglich und epistemisch bzw. explanatorisch auch hinreichend sind. ${ }^{82}$ Positionen mit wenig mehr epistemischer Skepsis berufen sich zuweilen auch auf einen weniger anspruchsvollen Erklärungsbegriff. ${ }^{83}$ Bei diesem gilt die Feststellung von kausalen Prozessen resp. von Korrelationen zwischen neurophysiologischen Prozessen und subjektiven Erlebnisberichten

81 Vgl. Atmanspacher, Harald: »Quantum Approaches to Consciousness«, in: The Stanford Encyclopedia of Philosophy, 2015, Online: http://plato.stanford.edu/archives/sum2015/ entries/qt-consciousness [zuletzt geprüft am 28.09.2018], Abschnitt 4.6 und 5.o.

82 Insofern kann bei epistemisch optimistischen Positionen mit der Terminologie Roths von einem »erkenntnistheoretischen Realismus« gesprochen werden. Dieser hält »objektives Wissen« für möglich, weil er davon ausgeht, dass Sachverhalte einer bewusstseinsunabhängigen Welt mindestens zum Teil so zu erkennen sind, wie sie »tatsächlich« sind. Vgl. Roth, Gerhard: Das Gehirn und seine Wirklichkeit. Kognitive Neurobiologie und ihre philosophischen Konsequenzen, Frankfurt am Main: Suhrkamp 1994, S. 340.

83 Z.B. haben Block und Stalnaker Levines Modell einer reduktiven Erklärung als unangemessen zurückgewiesen und es durch eine andere, weniger anspruchsvollere Konzeption von Erklärungen ersetzt. Vgl. dazu Block, Ned J. / Stalnaker, Robert: »Begriffsanalyse, Dualismus und die Erklärungslücke«, in: Begriff, Erklärung, Bewusstsein. Neue Beiträge zum Qualia-Problem, Pauen, Michael / Schütte, Michael / Staudacher, Alexander [Hrsg.], Paderborn: Mentis [zuerst 1999, engl.]/2007, S. 61-110. 
bereits als $>$ Erklärung des subjektiven Erlebens - oder sogar als Darlegung der $>$ Identität< von Erleben und neurophysiologischen Vorgängen. ${ }^{84}$

Ein epistemischer bzw. explanatorischer Optimismus im Hinblick auf das hard problem kann mithin auch mit einem reduktionstheoretischen Optimismus zusammenhängen. Bei letzterem wird davon ausgegangen, dass sich auch bei mentalen Phänomenen wie Bewusstsein zeigen lässt, dass sie $»$ nichts anderes $\ll^{85}$ als $»$ Dinge ${ }^{86}$ von einer bestimmten anderen Art sein sollen. ${ }^{87}$ Die Formel $>\mathrm{x}$ ist in Wirklichkeit nichts anderes als $\mathrm{y}<$ führt zu einer reduktionstheoretischen Identitätsbeziehung, die wir mit Searle als »Nichtsals-Beziehung ${ }^{88}$ bezeichnen können. Reduktionsoptimistische Positionen gehen davon aus, dass sich die Makroeigenschaft >Bewusstsein< durch eine Identitätsrelation mit bestimmten Mikroeigenschaften identifizieren lässt, die gewissermassen die >wesentliche Wirklichkeit der reduzierten Makroeigenschaft darstellen. Bei einem starken neurobiologischen Reduktionismus wird dies bspw. so verstanden, dass psychische Phänomene »[...] ihrem Wesen nach >nichts anderes sind als feuernde Nervenzellen $\ll .89$

Positionen, die in epistemischer Hinsicht eine Spur skeptischer sind, gehen im Hinblick auf das hard problem von "phänomenalen Begriffen ${ }^{90}$ aus, die zwar von physikalisch-funktionalen Begriffen unabhängig sind. ${ }^{91}$ Insofern sind phänomenale Begriffe mit physikalischen Begriffen nicht hinreichend beschreibbar. In solchen Positionen erklärt man sich die Besonderheit

84 Bei dieser Konzeption einer reduktiven Erklärung werden theoretische Identitätsbeziehungen und die explanatorische Leistungskraft theoretischer Identitätsaussagen vorausgesetzt, um damit, so die Annahme, die explanatorische Kraft einer reduktiven Erklärung von Bewusstsein zu erhöhen. Vgl. dazu Thöle, Bernhard: »Bewusstsein und Erklärungslücken«, in: Begriff, Erklärung, Bewusstsein. Neue Beiträge zum Qualia-Problem, Pauen, Michael / Schütte, Michael / Staudacher, Alexander [Hrsg.], Paderborn: Mentis 2007, S. 226. Vgl. ebenfalls Abschnitt 4.1.1.

85 So z.B. Dennett: Dennett, Daniel C.: »Lovely and Suspect Qualities«, 1991, Online: http:// cogprints.org/263/1/lovely\%26s.htm [zuletzt geprüft am 24.09.2018].

86 Searle, John R. / [Übers.: Gavagai, Harvey]: Die Wiederentdeckung des Geistes, München: Artemis-Verlag \& Winkler [zuerst 1992, engl.]/1993, S. 131. (Searle kritisiert dies.)

$87 \quad$ Vgl. dazu auch ebd.

88 A.a.O., S. 132.

89 Roth, Gerhard: Das Gehirn und seine Wirklichkeit. Kognitive Neurobiologie und ihre philosophischen Konsequenzen, Frankfurt am Main: Suhrkamp 1994, S. 285 [Hervorhebung im Original]. Nach Roth ist dies ein ontologisch-neurobiologischer Reduktionismus. Ein solcher sei in ausgearbeiteter Form jedoch selten zu finden. Vgl. dazu auch Paulsen, Friedrich: Einleitung in die Philosophie, Berlin: Wilhelm Herz [zuerst 1892]/19o1, S. 91.

9o Pauen, Michael / Schütte, Michael / Staudacher, Alexander [Hrsg.]: Begriff, Erklärung, Bewusstsein. Neue Beiträge zum Qualia-Problem, Paderborn: Mentis 2007, S. 16. Vgl. auch a.a.O., S. 22.

91 Vgl. a.a.O., S. 18. 
phänomenaler Begriffe aber damit, dass sie bspw. introspektiv generierte indexikalische Begriffe, ${ }^{92}$ Wiedererkennungsbegriffe ${ }^{93}$ sehr spezielle ErstePerson-Begriffe ${ }^{94}$ oder Vorstellungen über phänomenale Formen von Erleben seien..$^{95}$ Dies impliziere aber nicht, dass phänomenale Begriffe auf ein natürliches Phänomen hinweisen, zu dem wir keinen epistemischen Zugang hätten. ${ }^{96}$ Obwohl im Hinblick auf phänomenale Begriffe ein asymmetrischer epistemischer Zugang zur Erste-Person- gegenüber der DrittenPerson-Perspektive eingestanden wird, wird davon ausgegangen, dass »nichts Reales «97 mit dieser und anderen Eigenschaften phänomenaler Begriffe korrespondiere. ${ }^{98}$ Trotz eingestandener epistemischer Asymmetrie zwischen phänomenalen Begriffen der Erste-Person-Perspektive und physikalischen Begriffen glaubt man aber, dass es eine physikalische Erklärung dafür gebe, warum wir diese besonderen phänomenalen Begriffe besitzen. ${ }^{99}$ Positionen, die beim hard problem auf phänomenale Begriffe rekurrieren, sind demnach in epistemischer Hinsicht etwas skeptischer, als sie es in explanatorischer und reduktionstheoretischer Hinsicht sind.

Positionen des explanatorischen Kritizismus berufen sich auf das Problem der Erklärungslücke. ${ }^{100}$ Positionen des epistemischen Kritizismus finden sich bei dem Argumenttyp, der den epistemischen Zugang zu phänomenalen

92 Vgl. Lycan, William G.: Consciousness and experience, Cambridge, Massachusetts: MIT Press 1996.

93 Pauen, Michael / Schütte, Michael / Staudacher, Alexander [Hrsg.]: Begriff, Erklärung, Bewusstsein. Neue Beiträge zum Qualia-Problem, Paderborn: Mentis 2007, S. 17.

94 Tye, Michael: »Qualia«, in: The Stanford Encyclopedia of Philosophy, 2007, Online: http:// plato.stanford.edu/archives/fall2007/entries/qualia/ [zuletzt geprüft am 25.09.2018], Abschnitt 5.o.

95 Papineau, David: »Mind the Gap«, Philosophical Perspectives, [1998] Vol. 12: Language, Mind, and Ontology, S. 382 .

96 Vgl. dazu Tye, Michael: »Qualia«, in: The Stanford Encyclopedia of Philosophy, 2007, Online: http://plato.stanford.edu/archives/fall2007/entries/qualia/ [zuletzt geprüft am 25.09.2018], Abschnitt 5.o.

97 Balog, Katalin: »Phenomenal Concepts«, in: The Oxford Handbook of Philosophy of Mind, McLaughlin, Brian P. / Beckermann, Ansgar / Walter, Sven [Hrsg.], Oxford: Oxford University Press 2009, S. 299.

98 Eine asymmetrische Epistemologie der Ersten-Person- gegenüber der Dritte-PersonPerspektive wird bspw. von Balog, neben sechs anderen Eigenschaften von phänomenalen Begriffen aufgeführt. Ebd.

99 Vgl. dazu auch Chalmers, David J.: »Phänomenale Begriffe und die Erklärungslücke«, in: Begriff, Erklärung, Bewusstsein. Neue Beiträge zum Qualia-Problem, Pauen, Michael / Schütte, Michael / Staudacher, Alexander [Hrsg.], Paderborn: Mentis [zuerst 2006, engl.]/2007, S. 171, 172, 202 und 206.

100 Vgl. dazu Levine, Joseph: »Materialism and Qualia: The Explanatory Gap«, Pacific Philosophical Quarterly, [1983] Vol. 64, No. 4, S. 357. Vgl. dazu auch Abschnitt 4.1.1. 
Zuständen aus der Dritte-Person-Perspektive problematisiert, ${ }^{101}$ indem er auf die Asymmetrie des epistemischen Zuganges zu bewusstem Erleben, gegenüber intersubjektiv zugänglichen physikalischen Erkenntnisgegenständen hinweist. ${ }^{102}$

Demgegenüber ist der so genannte »Mysterianismus «, 103 vor allem in epistemischer und explanatorischer Hinsicht, noch skeptischer bzw. pessimistischer. Bewusstsein könnte nach diesem, in ontologischer Hinsicht, zwar auf Physikalisches zurückgeführt werden. ${ }^{104}$ Wie es aber auf Physikalisches reduziert werden könnte, ist epistemologisch gesehen absolut unzugänglich: die Verbindung zwischen Bewusstsein und neurophysiologischen Prozessen ist und bleibt für Menschen ${ }^{105}$ aufgrund einer konstitutionellen Limitation ${ }^{106}$ seines Verstandes »mysteriös «. ${ }^{107}$ McGinn begründet diese epistemologische Vorsicht mit dem Verweis darauf, dass schon bei grundlegenden physikalischen Fragen schwerwiegenden Probleme bestehen:

Physics too has its `hard p problems, as well as its >easy` problems. If nature as a whole, including the simple motions of matter, is shrouded in mystery, then we shouldn't be amazed to discover that mind is also. ${ }^{108}$

101 Vgl. dazu Jackson, Frank: »What Mary Didn't Know«, The Journal of Philosophy, [1986] Vol. 83, No. 5, S. 291-295. Vgl. dazu auch Abschnitt 4.1.2.

102 Vgl. z.B. Chalmers, David J.: »Reply to Mulhauser's Review of >The Conscious Mind««, 1996, Online: http://journalpsyche.org/files/oxaa32.pdf [zuletzt geprüft am 25.09.2018]. Vgl. auch Chalmers, David J.: The conscious mind. In search of a fundamental theory, New York: Oxford University Press 1996, S. 101-102.

103 Bspw. verwendet Seager diese Bezeichnung: »A yet weaker form of physicalism, sometimes called mysterianism, accepts ontological dependence but rejects all forms of epistemological dependence. On such a view, the way that mind depends upon its physical basis is irredeemably mysterious, inaccessible to human cognition «. Seager, William: »Emergentist Panpsychism «, Journal of Consciousness Studies, [2012] Vol. 19, No. 9-10, S. 22 [Hervorhebung E. E.].

104 Seager spricht deshalb im Hinblick auf den Mysterianismus von einer schwächeren Form des Physikalismus. Vgl. ebd. Diese Form des Physikalismus akzeptiert zwar eine epistemische, jedoch nicht zwingend eine ontologische Kluft zwischen Physikalischem und Phänomenalem. Vgl. dazu auch Alter, Torin / Nagasawa, Yujin: »What is Russellian Monism?«, Journal of Consciousness Studies, [2012] Vol. 19, No. 9-10, S. 87.

105 So konstatiert McGinn:»Human knowledge has its gaps and blind spots all over the place [...] «. McGinn, Colin: Basic structures of reality. Essays in meta-physics, New York: Oxford University Press 2011, S. 8.

106 Vgl. dazu auch McGinn, Colin: »Thought«, in: A companion to the philosophy of mind, Guttenplan, Samuel [Hrsg.], Malden, Massachusetts: Blackwell 2005, S. 588.

107 McGinn, Colin: Wie kommt der Geist in die Materie? Das Rätsel des Bewusstseins, München: Beck 2001, S. 172.

108 McGinn, Colin: Basic structures of reality. Essays in meta-physics, New York: Oxford University Press 2011, S. 7. 
Die tief verwurzelte (optimistische) Intuition, dass sich Bewusstsein unserem Erkenntnisvermögen explanatorisch erschliessen könnte, ist für McGinn sogar der Grund für das hard problem. ${ }^{109}$ Aus diesen Gründen kann man wohl den Mysterianismus als die >pessimistischste< Form des epistemischen und explanatorischen Kritizismus betrachten.

\subsection{Phänomenale Achse: Phänomenaler Realismus vs. phänomenaler Antirealismus}

Weil ich in dieser Arbeit mein Augenmerk auf das Desiderat phänomenaler Adäquatheit ${ }^{110}$ und folglich auf einen phänomenal adäquaten Qualia-Begriff lege, ist es wichtig, in ihr das hard problem im Lichte der Problemoptik der phänomenalen Achse zu analysieren. Da Erleben und Qualia Explananda darstellen, die zentral für ein künftiges Verständnis von Bewusstsein sind, ${ }^{111}$ kann die Wichtigkeit dieser Achse für eine Analyse des hard problem kaum überschätzt werden. Umgekehrt kann man folgern, dass Theorien des Geistes, die phänomenaler Adäquatheit ausweichen oder sie gar zu >eliminieren<versuchen, kaum vielversprechend sein können. Auf der phänomenalen Achse sind mir, stark vereinfacht, drei Kategorien von Zugangsweisen begegnet:

109 Dies begründet McGinn damit, dass wir einerseits einen »tief verwurzelten Hang« hätten, zu glauben, dass unser Erkenntnisvermögen Bewusstsein erklären bzw. erschliessen könne. Daraus entstehen auch die Bemühungen, Bewusstsein zu erklären. Wir wissen zwar einiges über physiologische Prozesse des Gehirns und dessen Struktur - und wir wissen, was sich, für uns wahrnehmbar auf der Oberfläche des Bewusstseins abspielt. Doch die Verbindung zwischen beiden verwirre uns nach wie vor und bleibe auch »mysteriös«. McGinn, Colin: Wie kommt der Geist in die Materie? Das Rätsel des Bewusstseins, München: Beck 2001, S. 172. Andererseits erwächst McGinn gemäss unser Staunen über das Bewusstsein, gerade aufgrund unseres nur oberflächlichen Zugangs zu diesem: »Die oberflächliche Erscheinung des Bewusstseins vermittelt keinerlei Eindruck von der ihm zu Grunde liegenden Struktur, also verharren wir in Erstaunen darüber, wie es möglich ist, dass Bewusstsein aus Gehirnaktivität resultiert«. Ebd.

110 Vgl. Stubenberg, Leopold: Consciousness and qualia, Amsterdam: John Benjamins 1998, S. 278 .

111 So konstatiert auch Tye: »The status of qualia is hotly debated in philosophy largely because it is central to a proper understanding of the nature of consciousness «. Tye, Michael: »Qualia«, in: The Stanford Encyclopedia of Philosophy, 2007, Online: http://plato. stanford.edu/archives/fall20o7/entries/qualia/ [zuletzt geprüft am 25.09.2018], Abschnitt einleitender Erläuterungen. Tye spricht zwar nur von der zentralen Bedeutung des Status von Qualia, damit gibt er (indirekt) zu, dass Qualia ein Explanandum darstellen: Denn etwas, dessen Status nicht klar ist, ist ein Phänomen, das einer Erklärung bedarf - eben ein Explanandum. 


\section{A.) Phänomenaler Antirealismus \\ B.) Phänomenaler Realismus \\ C.) Phänomenale Indifferenz}

A.) Positionen, welche bewusstem Erleben die >Existenzく bzw. Phänomenalem als Explanandum vollständig die Relevanz absprechen, nenne ich phänomenalen Antirealismus. Es ist mir bewusst, dass dies eine unübliche Verwendung des Begriffsteils >Realismus< ist, da sowohl ein epistemischer also auch ontologischer Realismus meistens als Gegenbegriffe zum Idealismus verwendet werden, weil beide von einer bewusstseinsunabhängigen Welt ausgehen. ${ }^{112}$ So scheint es vielleicht auf den ersten Blick widersprüchlich, wenn ich gerade jene Positionen, die Geistiges bzw. Phänomenales als etwas Nicht-Reales betrachten, als phänomenalen >Antirealismus< bezeichne. Die plakative Gegenüberstellung eines >Realismus`, im Sinne eines physikalistischmonistischen Realismus, der von einer bewusstseinsunabhängigen Wirklichkeit ausgeht, und einem idealistischen Standpunkt (bei dem explizit das Geistige den Wirklichkeitsvorrang hat) funktioniert jedoch nur, wenn beides in einem eingeengten Sinn verstanden wird. Wenn nun mitberücksichtigt wird, dass für jede und jeden letztendlich irgendetwas $>$ real $<$ ist, ${ }^{113}$ dann sind Positionen, die Phänomenales als etwas >Unwirkliches<, >Nicht-Reales< betrachten, als

112 Der ontologische Realismus geht von der Existenz einer bewusstseinsunabhängigen Welt aus, während epistemische Realisten noch zusätzlich daran glauben, dass die in jener Welt vorkommenden bewusstseinsunabhängigen Tatsachen epistemisch ganz und gar zugänglich sind bzw. dass objektives Wissen möglich ist.

Demgegenüber geht der ontologische Idealismus davon aus, dass die Annahme, dass die Welt auch dann existiere, wenn sie von keinem Menschen wahrgenommen wird, überflüssig oder gar widersinnig ist. Der epistemische Idealismus bestreitet wiederum, dass (bewusstseinsunabhängige) Objekte den Primat im Erkenntnisprozess haben bzw. dass objektives Wissen möglich ist, weil für ihn Wissen nur aus der Vernunft selber kommen kann. Vgl. dazu Gethmann, Carl F.: »Realismus (erkenntnistheoretisch)«, in: Enzyklopädie Philosophie und Wissenschaftstheorie. Band 3: P-So, Mittelstrass, Jürgen [Hrsg.], Stuttgart: Metzler 2004, S. 500-502. Vgl. ebenfalls Gethmann, Carl F.: »Realismus (ontologisch)《, in: Enzyklopädie Philosophie und Wissenschaftstheorie. Band 3: P-So, Mittelstrass, Jürgen [Hrsg.], Stuttgart: Metzler 2004, S. 502-504. Vgl. dazu auch Roth, Gerhard: Das Gehirn und seine Wirklichkeit. Kognitive Neurobiologie und ihre philosophischen Konsequenzen, Frankfurt am Main: Suhrkamp 1994, S. 340.

113 So lässt sich mit De Vries konstatieren: »Irgend etwas Wirkliches nimmt schliesslich jeder an, und wäre es auch das eigene `Selbst allein< $[\ldots] \ll$. De Vries, Josef: »Realismus«, in: Philosophisches Wörterbuch, Brugger, Walter [Hrsg.], Freiburg im Breisgau: Herder 1976, S. 316. Nach ihm zeugt es demnach von einem (zu) engen Wirklichkeitsbegriff bzw. Realitätsbegriff, wenn als >wirklich $<$ nur dasjenige gilt, welches von unserem Denken unabhängig ist: » Wirklich< ist vielmehr alles, dem Sein [...] zukommt, auch wenn dieses Sein, wie das unserer Akte und unserer äusseren Werke, von unserem Denken, Wollen und Tun abhängig ist«. A.a.O., S. 317 . 
Antipoden gegenüber denjenigen Positionen zu verstehen, für die Bewusstsein ein reales zu erklärendes Phänomen darstellt. In dieser Hinsicht bzw. im Lichte der phänomenalen Achse, können Konzeptionen, die von der >Unwirklichkeit< des Erlebens ausgehen, als Positionen mit einem spezifischen Antirealismus verstanden werden. Bei diesen Positionen handelt sich demnach nur um einen Anti-Realismus im Hinblick auf das Phänomenale. Wenn wir den Streit um das hard problem durch die Problemoptik der phänomenalen Achse betrachten, so geht es zwischen phänomenalem Realismus und phänomenalem Antirealismus um die Frage: Gibt es bewusstes Erleben? Der Begriffsteil >-Realismus< bezieht sich hier also darauf, welchen Phänomenen (k)ein »Realitätsstatus «114 zugeschrieben wird. Aus diesen Gründen bezeichne ich diejenigen Positionen, die davon ausgehen, dass es »in Wirklichkeit «115 kein bewusstes Erleben >gibt«, als >phänomenalen Antirealismus .

B.) Demgegenüber spricht Nagel von seinem »[...] Realismus bezüglich der subjektiven Domäne in all ihren Formen $[\ldots]{ }_{\ll}{ }^{116}$ Eine Position, die dem Erleben mindestens eine subjektive Realität zuschreibt, kann deshalb als »phänomenaler Realismus «117 bezeichnet werden. Im Gegensatz zum phänomenalen Antirealismus kann es zutreffen, dass sowohl explanatorisch (bzw. epistemisch) optimistische als auch explanatorisch pessimistische Theorien des Geistes bewusstes Erleben als Explanandum des hard problem betrachten. Das heisst, es spielt - im Hinblick auf die Anerkennung, dass Erleben etwas zu Erklärendes darstellt - keine Rolle, ob solche Theorien davon ausgehen, dass man Phänomenales erklären oder dass man es gar nie erklären könne.

114 So stellt Newen fest, dass den eliminativistischen Materialisten, egal welcher Prägung, gemeinsam ist, dass sie den Alltagsphänomenen wie z.B. Schmerzen, Überzeugungen, Furcht, bewussten Erlebnissen den »Realitätsstatus « absprechen und damit die Existenz dieser Phänomene leugnen. Vgl. Newen, Albert: Philosophie des Geistes. Eine Einführung, München: Beck 2013, S. 38 .

$115 \mathrm{Zu}$ dieser Begriffsverwendung vgl. z.B. Dennett, Daniel C.: »Why and How Does Consciousness Seem the Way it Seems? «, 2015, Online: http://open-mind.net/papers/whyand-how-does-consciousness-seem-the-way-it-seems [zuletzt geprüft am 24.09.2018], S. 3,8 und 10.

116 Nagel, Thomas: »Wie ist es, eine Fledermaus zu sein?«, in: Analytische Philosophie des Geistes, Bieri, Peter [Hrsg.], Weinheim: Beltz [zuerst 1974, engl.]/2007, S. 265 [Hervorhebung E. E.].

117 Dementsprechend wird die Bezeichnung »phänomenaler Realismus« von Block folgendermassen definiert: »Gemäss dem phänomenalen Realismus gilt: Wenn es sich wie Phänomenalität anfühlt, dann ist es Phänomenalität, was immer die materielle Basis sein mag $[. .$.$] «. Block, Ned J.: »Das schwierigere Problem des Bewusstseins«, in: Anatomie$ der Subjektivität. Bewusstsein, Selbstbewusstsein und Selbstgefühl, Grundmann, Thomas / Hofmann, Frank / Misselhorn, Catrin et al. [Hrsg.], Frankfurt am Main: Suhrkamp [zuerst 2002, engl.]/2005, S. 8o. Vgl. dazu auch weiter unten. 
Für Theorien des Geistes, die ich der Kategorie des phänomenalen Realismus zuordne, ist es im Hinblick darauf, dass bewusstes Erleben ein zu erklärendes Phänomen darstellt, auch eher zweitrangig, ob bzw. wie gut oder schlecht es zu unserem momentanen Erkenntnisstand physikalischer oder neuronaler Eigenschaften passen soll. Wenn also aus der sicherlich begrenzten Perspektive unseres noch zu entwickelnden Erkenntnisstandes die Meinung auftaucht, dass bewusstes Erleben vielleicht >nur< eine >Alltagsintuition< sein könnte, so verschwindet, durch die Umdeutung des Explanandums als »Täuschung «,118 wohl nicht, ${ }^{119}$ wie von phänomenalen Antirealisten vorgegeben, derart einfach das zu erklärende Phänomen. ${ }^{120}$

C.) Positionen, die bewusstes Erleben zwar vordergründig als Explanandum des hard problem betrachten, ihm jedoch durch Bedeutungsverschiebungen oder Umdeutungen ausweichen, subsumiere ich unter der Kategorie phänomenaler Indifferenz. Bei dieser Definition wird klar, dass sich Theorien des Geistes mit phänomenaler Indifferenz von Positionen, die ich >phänomenalen Antirealismus nenne, nur in kontinuierlichen Übergängen voneinander unterscheiden. Denn die von Positionen mit phänomenaler Indifferenz vorgenommenen Umdeutungen des zu Erklärenden Phänomens sind zuweilen derart stark, d.h. phänomenal inadäquat, dass sie in einigen Fällen das Explanandum bewussten Erlebens auf eine subtile Weise eliminieren, obschon sie sich selbst nicht als eliminativistisch betrachten würden. ${ }^{121}$

118 Dennett verwendet diesbezüglich z.B. den Begriff >Illusion $<$ Vgl. Dennett, Daniel C.: »Why and How Does Consciousness Seem the Way it Seems? «, 2015, Online: http://open-mind. net/papers/why-and-how-does-consciousness-seem-the-way-it-seems [zuletzt geprüft am 24.09.2018], S. 1 .

119 Denn es ist ebenfalls zweitrangig, dass unsere Urteile über den Gehalt phänomenalen Erlebens selbstverständlich falsch sein können. Das Explanandum des Erlebens verschwindet doch nicht einfach durch den Umstand, dass wir im Dunkeln eine Tomate fälschlicherweise als Apfel wahrnehmen können.

120 Mehr zu diesem Punkt in Abschnitt 9. An dieser Stelle sei nur einmal angemerkt, dass wenn wir bewusstes Erleben als eine >Täuschung umdeuten, denen Organismen unterliegen, das Explanandum immer noch darin bestünde, wie denn diese angebliche Täuschung überhaupt zustande kommen kann, wenn die dabei involvierten, >rein objektiv-physikalischen< Eigenschaften per Definition >nichts< erleben (weil Erleben ja nicht `existiert`). Wie könnten solche Organismen einer `Täuschung « unterliegen und von ihr den Eindruck bzw. das `Erlebnis $<$ haben, dass sie diese >erleben $<$ ?

121 Auf diese Problematik werde ich in Abschnitt 7.2 noch weiter eingehen. 